\title{
Best-Effort Cooperative Relaying
}

\author{
Nate Goergen, W. Sabrina Lin, K. J. R. Liu, and T. Charles Clancy
}

\begin{abstract}
Traditional cooperative communications consider dedicated-relays, while often such relays may not be available. In this paper, we consider wireless transceivers that relay signals in addition to their own primary communication mission. We consider a best-effort delivery policy, where a node is not obligated to devote energy to cooperatively relay signals, nor does it provide a guarantee of signal quality on retransmissions. Instead the relay sacrifices energy at its own discretion, with priority given to the primary communication mission. We consider one besteffort delivery problem: a system that transmits an additional relay signal within its original transmission energy budget while inducing minimal degradation to the primary-user's signal. To maintain this constraint, we consider the feasibility of reallocating energy from pilot signals used for channel estimation toward the relaying service, when channel conditions are stationary. We demonstrate that transmitter energy may be dynamically allocated between a relay component and a pilot component of the transmission using best-effort delivery. This power allocation is critical to system performance, since both the primary-user and the secondary-user may require pilot energy to correctly decode transmitted signals. Sub-optimal power allocation rules with respect to primary-user channel estimate mean-square error and pairwise error probability are derived.
\end{abstract}

Index Terms-Cooperative systems, MIMO, relays, wireless networks.

\section{INTRODUCTION}

C OOPERATIVE communication through the judicious use of relay-nodes has proven extremely advantageous in decreasing outage probabilities, [1], [2] and frame error rates [3] when the channel between the source and destination is of poor quality. Traditional relay schemes typically consider only nodes dedicated to relaying signals [4]. However, when a dedicated-relay is not available, nodes with their own communication mission must assist in relaying signals. We consider a relaying system where nodes provide relay services to a secondary-user in addition to their own primary communications, and the relay service is provided on a besteffort basis. Under the proposed scheme, a node with its own communication mission may also offer relay services while maintaining its original energy constraint. We extend the traditional three node relay problem considered in [4] to include the secondary-user node, and focus on the power allocation problem presented to the relay in this scenario. The best-effort relay system and the relationship between the secondary-user, or relay-user, and primary-user channels is depicted in Fig. 1, where the three node system of [4] is augmented to include the secondary-user.

Manuscript received April 23, 2010; revised August 15, 2010 and December 16, 2010; accepted January 3, 2010. The associate editor coordinating the review of this paper and approving it for publication was I. M. Kim.

The authors are with the Department of Electrical and Computer Engineering, University of Maryland, College Park, MD, 20742, USA (e-mail: goergen@umd.edu).

Digital Object Identifier 10.1109/TWC.2011.051211.100683

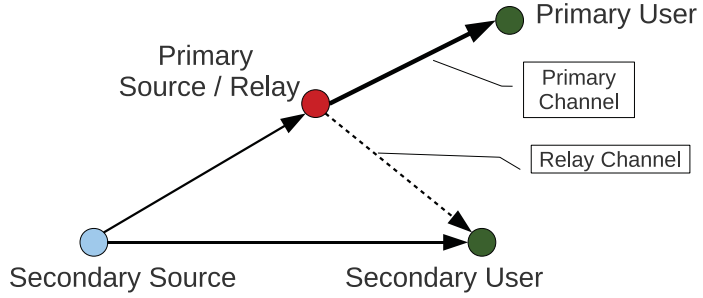

Fig. 1. System diagram of a best-effort relay system.

The terms primary-user and secondary-user have been used numerous times in the literature to describe precedence roles in cognitive radio and cooperative communications [5]. When a constrained resource is shared by a number of users, it is the primary-users that typically have first-right-of-access to the shared resource, and are given higher priority than the secondary-users. Our use of the terms in this work is similar.

As a motivating example for adaptive best-effort relaying we consider the scenario of stationary channel conditions for the primary-user channel, when less energy is required for channel estimation purposes. A number of studies have attempted to characterize the correlation of time-varying channel estimates, including the works of [6] and [7]. These studies have shown that in fixed and low-mobility scenarios consecutive channel estimates in multipath scenarios are highly correlated. Under these conditions the relay may choose to devote more power toward relaying services and less power toward pilot signals for the primary-user channel, according to the quality of service $(\mathrm{QoS})$ requirements of the primary-user.

When channel conditions require additional channel estimation energy, as is the case when the primary-user channel is undergoing change, the relay may choose to allocate more power toward the pilot component of the transmission for channel estimation purposes and less power for relay transmissions. The proposed best-effort delivery method introduces a unique power allocation problem in which the relay must select optimal power settings for the pilot and relayed signals. Power allocation for the relayed transmission is not guaranteed, and is rationed to aid the secondary-user only at the discretion of the primary-user. Thus, energy for the purpose of cooperative diversity through the relaying of signals is scavenged, when feasible, and the relayed transmissions are delivered on a besteffort basis.

Traditional pilot-aided channel estimation techniques, such as pilot symbol assisted modulation (PSAM), are discussed in [8]. Channel estimation schemes like PSAM create a composite signal consisting of two components: pilot signals used for channel estimation, and the user's data signal. To implement the relay service we instead consider a composite space-time code (STC) composed three components: the primary-user 
data signal, the pilot signal, and the relayed signal destined for the secondary-user. The density of the pilot signals and bandwidth resources for each component are assumed to be preallocated and constant, as we focus solely on the power allocation subproblem in this work. Also, we consider the case where the transmission power used for the primary-user data signal will remain constant in the composite signal; however the power devoted to the pilot signal component and the secondary-user component will be dynamically allocated.

While many pilot-embedding techniques have been proposed before, we formulate our best-effort delivery channel using the pilot-embedding framework proposed in [9], which generalizes how pilot signals may be embedded into data signals using a STC approach. With this embedding method, mutually orthogonal pilot and data signals are combined as a composite STC block before transmission. The STC approach is used as a general method for generating a sequence of symbols with periodically occurring pilot signals comprising a single block code, and allows for maximum flexibility in the design of pilot-aided transmissions for systems with one or more transmit antenna. The method easily extends to broadband signals such as OFDM, through the Space Time Frequency (STF) block code model [10]. Here, we investigate best-effort cooperative relaying using generic pilot embedding when the data-component of a data-bearing pilot block is reserved for relay transmissions.

Cooperative and Cognitive Radio (CR) systems can be complex systems faced with a number of resource allocation problems. Nodes that choose to cooperate must constantly ration resources such as transmission energy and bandwidth when these resources are limited [11], [12], [13]. While the rationing of bandwidth, for example, is an important resource allocation problem, in this paper we focus solely on the four node power allocation subproblem suggested by Fig. 1 and leave the extension of this subproblem to multi-node systems where bandwidth may also be rationed, as future work. The important cooperative system reciprocity issues of altruism and avariciousness are also not considered here; therefore, the motivations of the relay for aiding the secondary user, while interesting, are not applicable as this work focuses on cooperation once the decision to cooperate has been made.

In this paper, we discuss a number of optimization problems that arise when considering the best-effort delivery of signals, and derive sub-optimal power allocation policies with respect to the upper-bound mean squared error (MSE) of the channel estimate, and the pairwise error probability (PEP) of the primary-user. The power allocation problem and general feasibility of best-effort delivery are considered using these two QoS criterion. We extend our work in [14] to consider the MSE-based power allocation rule and the total bit error rate (BER) of the system, comparing the performance of both allocation rules in greater detail. Additionally, sub-optimal power allocation considering the aggregate capacity of both the primary and secondary users is discussed.

This paper is structured as follows: Section II generalizes data-bearing pilot frameworks (DBPF) and briefly introduces previous power-allocation work with secondary-channels. In Section III the best-effort relay problem is discussed, and a model for analytic system design is presented. Sub-optimal power allocation policies, with respect to the primary-user, are derived taking the upper-bound channel estimate MSE and PEP into consideration. In Section IV simulation results are presented, and in Section V we give some concluding remarks.

\section{System Model and Problem Formulation}

We briefly describe the channel model used in our besteffort delivery scheme. We assume all nodes are MIMO systems with $L_{t}$ transmit antenna(s) and $L_{r}$ receive antenna(s) and use a Space Time (ST) block scheme, where the block transmitted at time index $t$ is described by matrix $\mathbf{U}(t)$ of size $L_{t} \times M$. The ST block $\mathbf{U}(t)$ is a composite signal composed three components: the primary-user data signal, the pilot signal, and the relayed signal destined for the secondaryuser. The composite signal is transmitted across all $L_{t}$ transmit antennas in $M$ time slots, and is broadcast to both the primary and secondary users. The received block $\mathbf{Y}(t)$ expressed in matrix form is

$$
\mathbf{Y}(t)=\mathbf{H}(t) \mathbf{U}(t)+\mathbf{N}(t)
$$

with the channel coefficient matrix $\mathbf{H}(t)$ of size $L_{r} \times L_{t}$ describing the channel conditions experienced by the block at time $t$. The channel noise $\mathbf{N}(t)$ is modeled as complex white Gaussian noise with zero mean and variance $\left(\sigma^{2} / 2\right) \mathbf{I}_{\left(L_{r} \times M\right)}$. We assume Rayleigh block fading for the elements of $\mathbf{H}(t)$, where the entries of $\mathbf{H}(t)$ are independent zero mean complex Gaussian random variables that remain constant over each symbol block. The channel estimation problem is to estimate $\mathbf{H}(t)$ and recover the original ST block $\mathbf{U}(t)$, where the channel estimate is computed from the pilot / training signals. The pilot or training signal is embedded in the original transmission $\mathbf{U}(t)$, using a generalized embedding scheme to be described as follows.

We now briefly describe the pilot-embedding framework presented in [9], which provides the edifice for the design of $\mathbf{U}(t)$. The ST block to be transmitted is given as

$$
\mathbf{U}(t)=\mathbf{D}(t) \mathbf{A}+\mathbf{P},
$$

where $\mathbf{P} \in \mathcal{R}^{L_{t} \times M}$ is the pilot matrix, ST block databearer matrix $\mathbf{D}(t) \in \mathcal{C}^{L_{t} \times N}$, and data-projection matrix $\mathbf{A} \in$ $\mathcal{R}^{N \times M}$. Here, $N$ is the number of time slots reserved for data transmission, while time slots $M-N, N<M$ are reserved for the embedded pilot signals and the relayed signal for the secondary-user. The pilot-embedding structures discussed in [9] have a bandwidth efficiency which is proportional to $\left(M-L_{t}\right) / M$ for the case $M=N+L_{t}$. The ST channel matrix is assumed to follow the constraint $E[\|\mathbf{H}(t)\|]^{2}=L_{t}$, or constant energy under the Frobenius norm. The salient point of the data-bearing framework is that pilot-embedding schemes may be generalized through the superposition of the databearing structure $\mathbf{D}(t) \mathbf{A}$ and the pilot matrix $\mathbf{P}(t)$. The dataprojection and pilot matrix satisfy the following properties:

$$
\begin{array}{ll}
\mathbf{A P}^{T}=\mathbf{0} \in \mathcal{R}^{N \times L_{t}}, & \mathbf{P A}^{T}=\mathbf{0} \in \mathcal{R}^{L_{t} \times N}, \\
\mathbf{A A}^{T}=\beta \mathbf{I} \in \mathcal{R}^{N \times N}, & \mathbf{P P ^ { T }}=\alpha \mathbf{I} \in \mathcal{R}^{L_{t} \times L_{t}} .
\end{array}
$$

where $\beta$ is the real-valued power of the relayed signal, and $\alpha$ is a real-valued power coefficient of the pilot portion of the signal. The $\alpha$ coefficient will become an important 
parameter in the analysis to come, as it represents the fraction of divertable power that is retained for pilot signals. Since the power allocated to the relay and pilot signals is allocated dynamically, it is assumed that both the primary and secondary user receivers employ either independent gain control for each signal, or convey the value of $\alpha$ used for each block to the receivers using a control channel signal that is not considered here.

We assume that the number of time slots $M$ in the ST transmission $\mathbf{U}(t)$ remains constant, and the number of time slots used for data transmission $N$ also remains constant. Using this criteria the number of time slots available for besteffort transmissions also remains constant.

The properties (3) of the data-projection matrix $\mathbf{A}$ and $\mathbf{P}$ essentially allow A to project the data component $\mathbf{D}(t)$ onto the orthogonal subspace of the pilot matrix $\mathbf{P}$, allowing for signal demodulation by means of a Maximum Likelihood (ML) receiver. These properties imply that $\operatorname{Rank}(\mathbf{A})=N$, $\operatorname{Rank}(\mathbf{P})=L_{t}$, and the number of time slots $M$ required of the ST symbol $\mathbf{U}(t)$ is $M=\operatorname{Rank}(\mathbf{A})+\operatorname{Rank}(\mathbf{P})$. The pilot structures of (3) operate under a power constraint

$$
\beta=P_{p}-\alpha,
$$

with $P_{p}$ being the original normalized block transmission power of the node. According to (4) the power allocated to the composite signal consisting of the pilot plus secondaryuser data signal is equal to the power of the original node transmission. To understand the behavior of the power allocation term $\alpha$ in terms of secondary-user performance, we first note that the normalized block power may be expressed as

$$
\begin{aligned}
P_{p} & =\frac{E\left[\|\mathbf{U}(t)\|^{2}\right]}{L_{t}}=\frac{E\left[\|\mathbf{D}(t) \mathbf{A}\|^{2}\right]}{L_{t}}+\frac{E\left[\|\mathbf{P}\|^{2}\right]}{L_{t}}, \\
& =\beta+\alpha=1 .
\end{aligned}
$$

The signal at the receiver becomes

$$
\mathbf{Z}(t)=\mathbf{H}(t)(\mathbf{D}(t) \mathbf{A}+\mathbf{P})+\mathbf{N}(t)
$$

Three basic structures are discussed in [9] for the design of A and $\mathbf{P}$, including the Time-Multiplexed (TM) structure which generalizes the previous PSAM pilot embedding techniques. The TM structure, which will be used later in simulation, is given as

$$
\begin{aligned}
& \mathbf{A}=\sqrt{\beta}\left[\mathbf{0}_{\left(N \times L_{t}\right)} ; \mathbf{I}_{(N \times N)}\right] \\
& \mathbf{P}=\sqrt{\alpha}\left[\mathbf{I}_{\left(L_{t} \times L_{t}\right)} ; \mathbf{0}_{\left(L_{t} \times N\right)}\right],
\end{aligned}
$$

Since the relay node must make the primary communication mission top priority, we adopt a conservative policy for prioritizing transmissions. Therefore, upper-bounds are used to ensure that the primary-user QoS constraint is met even in worst-cast channel conditions. The Chernoff upperbound pairwise error probability (PEP) with respect to an independent Rayleigh distributed channel is expressed by [9]

$P(\mathbf{d} \rightarrow \mathbf{e})_{\hat{\mathbf{H}}(t)} \leq\left(\prod_{i=1}^{L_{\Delta}} \lambda_{i}\right)^{-L_{r}}\left(\frac{\sigma_{Q}^{2}}{\frac{4}{N}\left(\frac{N}{\beta}+\frac{L_{t}}{\alpha}\right) \sigma^{2}}\right)^{-L_{\Delta} L_{r}}$

where $\lambda_{i}$ are the eigenvalues of the code error matrix defined as $C_{p, q}=\mathbf{x}_{q}^{H} \mathbf{x}_{p}$ and $\mathbf{x}_{p}=\left(d_{1}^{P}-e_{1}^{P}, \cdots, d_{N}^{P}-e_{N}^{P}\right)^{T}$ is the pairwise difference between the code and the erroneously detected code. In (8), $\sigma_{Q}^{2}=1+\left(\sigma^{2} / \alpha\right)$ represents the variance of an element in the estimated channel coefficient matrix $\hat{\mathbf{H}}(t)$, and $L_{\Delta}$ is the rank of the ST code with maximum achievable rank $L_{t}$. This PEP expression will be used in the next sections to analyze performance of the primary-user signal and the secondary-user signal, when adapting to different channel conditions. Another important result is the pair-wise error probability for the maximum-likelihood receiver, where the channel state information $\mathbf{H}(t)$ is known exactly. We refer the reader to [15] for this result, and the derivation of channel estimate MSE.

Relay-aided cooperative communication is achieved when a signal transmitted by a source node is received by one or more intermediate relay-nodes, who in turn retransmit the source's signal to the destination. Such systems offer performance advantages in terms of spatial diversity and power gain [1]. In the relaying scheme known as Decode-and-Forward (DF), a relay first receives and then decodes the signal from the source node before retransmitting the signal to the destination. It may be shown that the tight upper-bound PEP for the DF dedicatedrelay scheme in sufficiently high SNR can be expressed as

$$
P_{s D F} \approx \frac{N_{0}^{2}}{b^{2}} \cdot \frac{1}{P_{1} \delta_{s, d}^{2}}\left(\frac{A^{2}}{P_{1} \delta_{s, r}^{2}}+\frac{B}{P_{2} \delta_{r, d}^{2}}\right),
$$

where $N_{0}^{2}$ is the receiver noise power, $\delta_{s, d}^{2}, \delta_{s, r}^{2}$, and $\delta_{r, d}^{2}$ are the instantaneous channel gains between the source and destination, the source and relay, and the relay to destination respectively, and $A, B$, and $b$ are modulation specific constants defined in [16]. For example, if QPSK is employed $A, B$, and $b$ take on particular values, and if 16QAM is employed $A$, $B$, and $b$ take on another set of values. The real-valued terms $P_{1}$ and $P_{2}$ represent the optimal power allocations used for transmission by the source and the dedicated DF relay, and are given respectively as

$$
\begin{gathered}
P 1=\frac{\delta_{s, r}+\sqrt{\delta_{s, r}^{2}+8\left(A^{2} / B\right) \delta_{r, d}^{2}}}{3 \delta_{s, r}+\sqrt{\delta_{s, r}^{2}+8\left(A^{2} / B\right) \delta_{r, d}^{2}}} P, \\
P 2=\frac{2 \delta_{s, r}}{3 \delta_{s, r}+\sqrt{\delta_{s, r}^{2}+8\left(A^{2} / B\right) \delta_{r, d}^{2}}} P .
\end{gathered}
$$

We will compare the fixed power allocations for the dedicatedrelay DF scheme, (9) and (10), to the dynamic QoS-based allocation rules presented in Section III.

The power optimization problem can be formulated as finding the minimum pilot power factor $\alpha$ that maintains a QoS level with respect to the primary-user, denoted $G_{p}$, according to some QoS rule $G(\alpha, \eta)$. That is minimizing $\alpha$ subject to

$$
G(\alpha, \eta) \geq G_{p}, \quad 0 \leq \alpha \leq 1,0 \leq \eta \leq 1
$$

where the coefficient $\eta$ represents the influence of previous primary-channel state information when equalizing the current primary-user block, and will be defined in Section III. One may also interpret $\eta$ as the confidence of previous channel state information in modeling current channel state. If channel conditions have deviated from the previous channel estimate 
and there is less confidence in previous channel state information, the receiver will update its channel estimate using the pilot signals embedded in the transmission. The estimation of channel variances and $\eta$ may be performed by the receiver and communicated to the transmitter through a side control channel; however the discussion of this control channel is outside the scope of this work.

\section{ANALYSIS}

In the following subsections we present the pairwise error probability, MSE for the channel estimate, and power allocations for the best-effort relaying system. Since the power allocations presented are derived with respect to upper-bound system pairwise error probability and MSE, to ensure that these QoS criterion are unconditionally maintained for system users, the following allocations are sub-optimal.

\section{A. PEP and MSE for the Best-Effort Delivery Policy}

In this section we consider the PEP and channel estimate MSE experienced by the primary and secondary users using the system model presented in Section II. Let us consider the case where channel between the relay and the primary-user, depicted as the bold/solid line in Fig. 1, is stationary over at least two consecutive blocks, and the primary-user is able to detect this event. Such conditions may occur between fixed or immutable nodes, and in this motivating example we assume that transmission of additional pilot signal energy will not dramatically alter or improve the receiver's channel estimate. When the primary-user channel estimate $\hat{\mathbf{H}}_{p}(t)$ has perfectly estimated the channel $\mathbf{H}_{p}(t)$, that is

$$
\hat{\mathbf{H}}_{p}(t)=\mathbf{H}_{p}(t),
$$

the Chernoff upper-bound PEP for the primary-user becomes

$$
P(\mathbf{d} \rightarrow \mathbf{e})_{\mathbf{H}_{p}(t)} \leq\left(\prod_{i=1}^{L_{\Delta_{p}}} \lambda_{i_{p}}\right)^{-L_{r_{p}}}\left(\frac{P_{p}}{4 \sigma_{p}^{2}}\right)^{-L_{\Delta_{p}} L_{r_{p}}},
$$

where $L_{\Delta_{p}}$ is the rank of the primary-user's channel $\mathbf{H}_{p}(t)$, $\lambda_{i_{p}}$ are the eigenvalues of the primary-channel, $L_{r_{p}}$ are the number of receive antennas used by the primary-user, and $P_{p}$ is the normalized power used by the primary/relay-node for transmission. The Chernoff upper-bound PEP for the secondary-user can be expressed as

$$
\begin{aligned}
& P(\mathbf{d} \rightarrow \mathbf{e})_{\hat{\mathbf{H}}_{s}(t)} \leq \\
& \left(\prod_{i=1}^{L_{\Delta_{s}}} \lambda_{i_{s}}\right)^{-} L_{r_{s}}\left(\frac{1+\frac{\sigma_{s}^{2}}{\alpha}}{\frac{4 \sigma_{s}^{2}}{N}\left(\frac{N}{P_{p}-\alpha}+\frac{L_{t_{s}}}{\alpha}\right)}\right)^{-L_{\Delta_{s}} L_{r_{s}}},
\end{aligned}
$$

where $L_{\Delta_{s}}$ is the rank of the the channel estimate $\hat{\mathbf{H}}_{s}(t)$ between the the relay and the secondary-user, $\lambda_{i_{s}}$ are the eigenvalues of the secondary-channel, and $L_{r_{s}}$ are the number of receive antennas used by the secondary-user. We note that in this situation the node is fullfilling primary mission obligations while concurrently acting as a relay for the secondary-user. Thus the channel $\mathbf{H}_{s}$ is analogous to $\delta_{r, d}$ and its MIMO

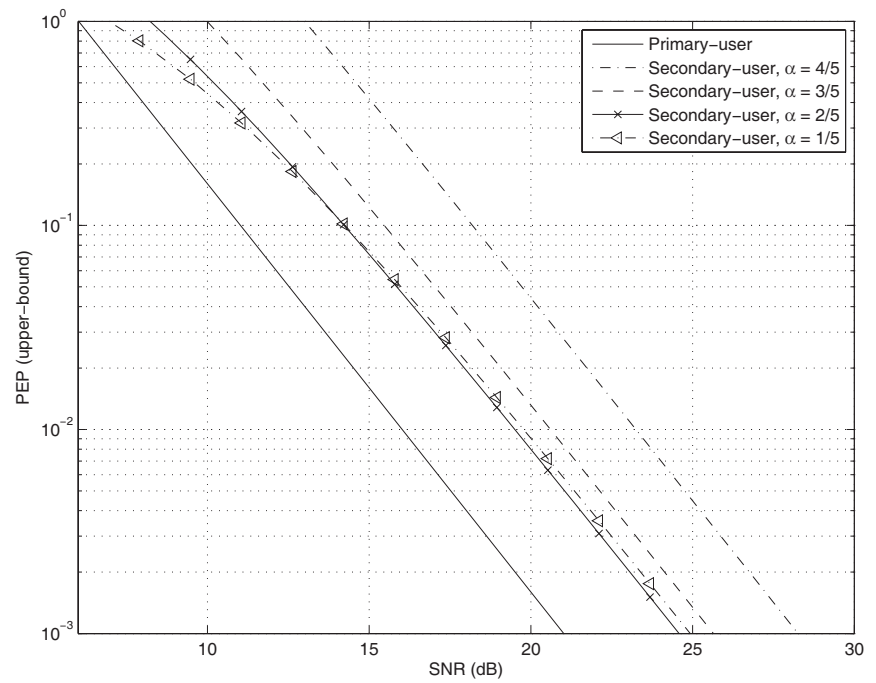

Fig. 2. Theoretical upper-bound of PEP of primary and secondary-user for values of $\alpha$.

representation $\mathbf{H}_{r, d}$, referring to the dedicated-relay notation used in Section II.

Under the proposed best-effort delivery policy, power for channel estimation purposes is diverted by the primary-user toward relay transmissions for the secondary-user. It should be noted that the secondary-user also requires energy for proper channel estimation, thus there exists a performance tradeoff for the secondary-user as power to the pilot signals is decreased. A plot of the PEP upper-bound for the secondary-user vs SNR, with respect to the PEP of the primary-user, for various values of pilot power allocations $\alpha$ is depicted in Fig. 2 for the conditions $L_{r_{p}}=L_{r_{s}}=2$ with $N=2$ time slots and $L_{t_{s}}=2$, for unit gain channels $\Delta_{p}=\Delta_{s}=1$ and $\lambda_{i_{s}}=\lambda_{i_{p}}=1$. In this figure, SNR is defined as $S N R=P_{p} L_{t_{p}} / \sigma_{p}^{2}$, as suggested by (25) and (28). From this figure we note that as the value of $\alpha$ decreases the PEP for the secondary-user increases; however the secondary-user is always at a disadvantage with respect to the primary-user, when both users experience identical channel conditions.

We now look at the power allocation problem between the pilot-part and relay-part of the proposed scheme, and how this criteria may change with respect to the needs of the primaryuser. This model will be used in the coming subsections to derive sub-optimal power allocation rules. By substituting the power constraint (5) into the PEP mismatch equation given in [9], the power optimization problem with respect to the secondary-user becomes

$$
\min _{\alpha} \ln \left(\frac{\left(N-L_{t}\right) \alpha+P_{p} L_{t_{p}}}{\left(\alpha+\sigma^{2}\right)\left(P_{p}-\alpha\right)}\right) .
$$

Since the primary-user may use its prior channel estimate when current channel conditions do not warrant re-estimation of the channel, the MSE for the primary-user does not depend directly on $\alpha$ because pilot signals are ignored in this case. However, for each block transmission there is a chance that channel conditions will significantly change, requiring the primary-user to update its channel estimate using the pilot signal embedded in the transmission. We model this scenario in simple probabilistic terms as a two-state model, with 
channel state $\nu$ given as

$$
\nu= \begin{cases}0, & \hat{\mathbf{H}}_{p}(t-1)=\hat{\mathbf{H}}_{p}(t)=\mathbf{H}_{p}(t) \\ 1, & \hat{\mathbf{H}}_{p}(t-1) \neq \mathbf{H}_{p}(t) .\end{cases}
$$

We define the model parameter $\eta$ such that

$$
\eta=P(\nu=0)=1-P(\nu=1),
$$

thus $\eta$ is the probability that the previous channel state information is sufficient for equalizing the current block, and $1-\eta$ is the probability that the primary-user must update its channel estimate based on the pilot signals present. In reality the estimates $\hat{\mathbf{H}}_{p}(t-1)$ and $\hat{\mathbf{H}}_{p}(t)$ will never perfectly match current channel conditions $\mathbf{H}_{p}(t)$; therefore, in practice the assertion of estimates being a 'perfect match' can be described in terms of being within some extremely small threshold of error from $\mathbf{H}_{p}(t)$, and that this error is negligible.

In state $\nu=0$, the primary-channel is considered stationary. Thus the channel estimate for the current code remains unchanged from the previous channel estimate, and we assume that the previous channel estimate has converged to match the current channel state. The channel estimate MSE for perfectly estimated channel conditions is given as

$$
E\left[M S E_{p}(t \mid \nu=0)\right]=\operatorname{tr}\left\{\operatorname{Cov}\left[\tilde{\mathbf{h}}_{p}(t)\right]\right\}=\sigma_{p}^{2} L_{t_{p}} L_{r_{p}},
$$

where $\tilde{\mathbf{h}}_{p}(t)$ is the vectorization of $\hat{\mathbf{H}}_{p}(t)$ discussed in [9]. The primary-user PEP is given as

$$
P(\mathbf{d} \rightarrow \mathbf{e} \mid \nu=0)_{\mathbf{H}_{p}(t)} \leq\left(\prod_{i=1}^{L_{\Delta_{p}}} \lambda_{i_{p}}\right)^{-L_{r_{p}}}\left(\frac{P_{p}}{4 \sigma_{p}^{2}}\right)^{-L_{\Delta_{p}} L_{r_{p}}}
$$

When the channel state is $\nu=1$, channel conditions for the primary-user have changed substantially, requiring the receiver to update its channel estimate $\hat{\mathbf{H}}_{p}(t)$. In this state the receiver will experience an MSE and PEP from (14) expressed as

$$
\begin{aligned}
& E\left[M S E_{p}(t \mid \nu=1)\right]=\operatorname{tr}\left\{\operatorname{Cov}\left[\tilde{\mathbf{h}}_{p}(t)\right]\right\}=\frac{\sigma_{p}^{2} L_{t_{p}} L_{r_{p}}}{\alpha}, \\
& P(\mathbf{d} \rightarrow \mathbf{e} \mid \nu=1)_{\hat{\mathbf{H}}_{p}(t)} \leq \\
& \left(\prod_{i=1}^{L_{\Delta_{p}}} \lambda_{i_{p}}\right)^{-L_{r_{p}}}\left(\frac{1+\frac{\sigma_{p}^{2}}{\alpha}}{\frac{4 \sigma_{p}^{2}}{N}\left(\frac{N}{P_{p}}+\frac{L_{t_{p}}}{\alpha}\right)}\right)^{-L_{\Delta_{p}} L_{r_{p}}},
\end{aligned}
$$

where parameters $L_{\Delta_{p}}, \lambda_{i_{p}}, L_{r_{p}}$, and $\sigma_{p}^{2}$ for the primary-user in state $\nu=1$ are defined similarly to those of the secondaryuser in (14) and are independent from those of the secondaryuser except for the common factors $L_{t_{p}}, \alpha$, and $P_{p}$. We notice that while energy allocated to the data part of the primaryuser's signal remains constant in the best-effort scheme, the PEP expression for a primary-user using pilot signals to reestimate channel conditions is dependent on $\alpha$ since both the primary and secondary-users must use the energy in these signals for channel estimation when $\nu=1$. The expected MSE for the primary-user defined by the two-state model becomes

$$
\begin{aligned}
E\left[M S E_{p}(t)\right] & =M S E_{p}(t \mid \nu=0) P[\nu=0] \\
& +M S E_{p}(t \mid \nu=1) P[\nu=1] \\
& =\frac{\sigma_{p}^{2} L_{t_{p}} L_{r_{p}}}{\alpha}(\eta \alpha+1-\eta) .
\end{aligned}
$$

The signal power of the pilot component for the current block is determined by the transmitter's selection of $\alpha$ for that block transmission. The MSE and PEP for the secondaryusers will be similar to (20) and (21) respectively; however the parameters $\sigma_{p}^{2}, L_{r_{p}}, \hat{\mathbf{H}}_{p}(t), \lambda_{i_{p}}, \lambda_{\delta_{p}}$ become the parameters of the secondary-channel, $\sigma_{s}^{2}, L_{r_{s}}, \hat{\mathbf{H}}_{s}(t), \lambda_{i_{s}}$, and $\lambda_{\delta_{s}}$.

\section{B. Sub-optimal Power Allocation with Respect to Channel Estimate MSE}

We now consider the power optimization problem with respect to a QoS rule limiting the MSE of the primary-user channel estimate. Since channel estimation performance for the primary-user (22) depends on pilot signal energy, proper selection of $\alpha$ to maintain a minimum QoS for the primaryuser is critical. For comparison, we first consider the suboptimal power allocation considering only the relay signal to the secondary-user, i.e. when the primary-user is omitted from Fig. 1. The sub-optimal power allocation considering only the relay transmission, $\alpha^{*}$, can be found by taking the derivative of (15) and setting to zero, i.e

$$
\alpha^{*}= \begin{cases}\frac{P_{p}-\sigma_{p}^{2}}{2} & N=L_{t_{p}} \\ \frac{P_{p} L_{t_{p}}-\sqrt{P_{p} N\left(P_{p} L_{t_{p}}+\sigma_{p}^{2}\left(L_{t_{p}}-N\right)\right)}}{L_{t_{p}}-N} & N \neq L_{t_{p}},\end{cases}
$$

where $\alpha^{*}$ is the sub-optimal power allocation considering only the secondary-user, $P_{p}$ is the power allocated to pilot plus relay transmissions, $L_{t_{p}}$ is the number of transmit antenna which is the same for all transmissions, and $\sigma^{2}$ is the channel variance experienced by the pilot signal. Thus, the sub-optimal power allocation rule considering only the secondary-user is simply $\alpha_{s}^{*}=\alpha^{*}$. We now derive the sub-optimal power allocation rule considering a minimum level of QoS for the primary-user only, denoted $\alpha_{p}^{*}$. The primary-user maximum MSE threshold condition, derived according to the two-state model, is given as

$$
M S E_{p}=\frac{\sigma_{p}^{2} L_{t_{p}} L_{r_{p}}}{\alpha}(\eta \alpha+1-\eta) \leq T_{p},
$$

where $T_{p}$ is the maximum channel estimation error allowed for the primary-user. It is worth noting that for the case $N \neq L_{t}$, the sub-optimum solution for the pilot-power allocation factor $\alpha^{*}$ in (23) exists if and only if $S N R \geq\left(N-L_{t_{p}}\right)$, where $S N R=P_{p} L_{t_{p}} / \sigma_{p}^{2}$. In Section IV we consider the case where $L_{t_{p}}=N=2$, thus for the sake of exposition we will consider the case of $N=L_{t_{p}}$ in our analysis here. We substitute the sub-optimal power allocation $\alpha=\frac{P_{p}-\sigma_{p}^{2}}{2}$ from (23) into (24) producing the SNR constraint

$$
S N R_{p} \geq L_{t_{p}}^{2} L_{r_{p}}\left(\frac{2-2 \eta-\sigma_{p}^{2} \eta+\frac{T_{p}}{L_{t_{p}} L_{r_{p}}}}{T_{p}-L_{t_{p}} L_{r_{p}} \eta \sigma_{p}^{2}}\right),
$$

where $0 \leq \alpha \leq 1$, and $S N R_{p}$ is the signal to noise ratio of the primary user as a function of the MSE threshold, $T_{p}$, and the noise variance of the primary-user's channel, $\sigma_{p}^{2}$. Using (24) and (25) and solving for $\alpha$, the sub-optimal allocation for the primary-user according to the maximum channel estimate MSE threshold rule, $\alpha_{p_{M S E}}^{*}$, considering the $S N R_{p}$ constraint and $M S E_{p} \leq T_{p}$ becomes

$$
\alpha_{p_{M S E}}^{*}=\frac{(\eta-1)\left(\gamma+T_{p} \pm \sqrt{\xi}\right)}{\eta\left(\gamma-T_{p} \pm \sqrt{\xi}\right)}
$$




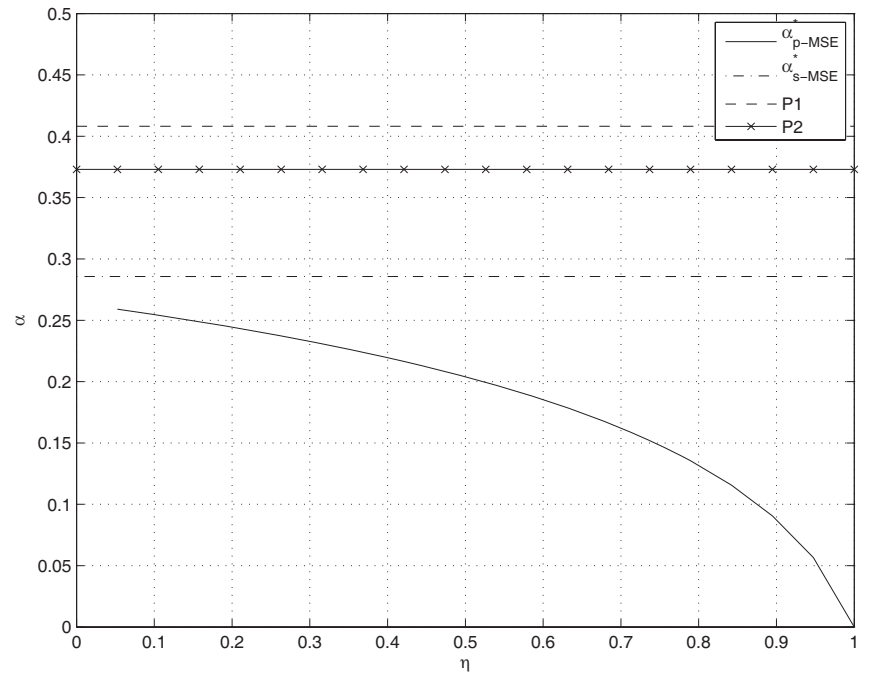

Fig. 3. $\alpha_{p_{M S E}}^{*}, \alpha_{s_{M S E}}^{*}, P 1$ and $P 2$ vs. $\eta$.

with

$$
\begin{aligned}
\gamma & =L_{t_{p}} L_{r_{p}}\left(P_{p} \eta-2 \eta+2\right), \\
\xi & =4 L_{t_{p}} L_{r_{p}} \eta\left(L_{t_{p}} L_{r_{p}}\left(\frac{P_{p} \eta}{4}+P_{p}-P_{p} \eta+\frac{1}{\eta}+\eta-2\right)\right. \\
& \left.+T_{p}\left(\frac{1}{\eta}-\frac{P_{p}}{2}-1\right)\right)+T_{p}^{2} .
\end{aligned}
$$

We observe from (26), that the power allocation rule $\alpha_{p_{M S E}}^{*}$ is valid only when $\xi$ is positive. For the sake of exposition, we will only consider the case when $\xi>0$ here, and will assume that $\alpha$ will take on the values of either zero or one otherwise. Also, since $\alpha$ must be positive and in the range $0 \leq \alpha \leq 1$, only the positive values of (26) will be considered as valid allocations.

While the best-effort policy relay-node will optimize its parameters with respect to its own transmissions before secondary-user requirements are considered, for comparison the optimum pilot-power allocation considering only the secondary-user under the same maximum channel estimate MSE threshold criterion for the case $N=L_{t}$ is simply

$$
S N R_{s} \geq L_{t_{p}}+\frac{2 L_{t_{p}}^{2} L_{r_{s}}}{T_{s}}
$$

with QoS threshold $T_{s}$, secondary-user signal to noise ratio $S N R_{s}$, and sub-optimum value of $\alpha_{s}$ satisfying (28) under the maximum MSE rule, $\alpha_{S M S E}{ }^{*}$, becomes

$$
\alpha_{s M S E}{ }^{*}=\frac{L_{t_{p}} L_{r_{s}} P_{p}}{T_{s}+2 L_{t_{p}} L_{r_{s}}}, \quad 0 \leq \alpha \leq 1 .
$$

We note that the MSE experienced by the secondary-user is a function of $\alpha$ but not $\eta$, and is expressed as

$$
M S E_{s}=\frac{\sigma_{s}^{2} L_{t_{p}} L_{r_{s}}}{\alpha} \leq T_{s}, \quad 0 \leq \alpha \leq 1 .
$$

To demonstrate behavior of the best-effort power allocation policy, in Fig. 3 we plot the values of $\alpha_{p_{M S E}}^{*}$ and $\alpha_{s_{M S E}}^{*}$ with respect to $\eta$, according to (26) and (29) respectively. Here we choose $L_{t_{p}}=L_{r_{p}}=L_{r_{s}}=2, P_{p}=P_{s}=1$, and the select $T_{s}=T_{p}=7$ for the MSE thresholds. These

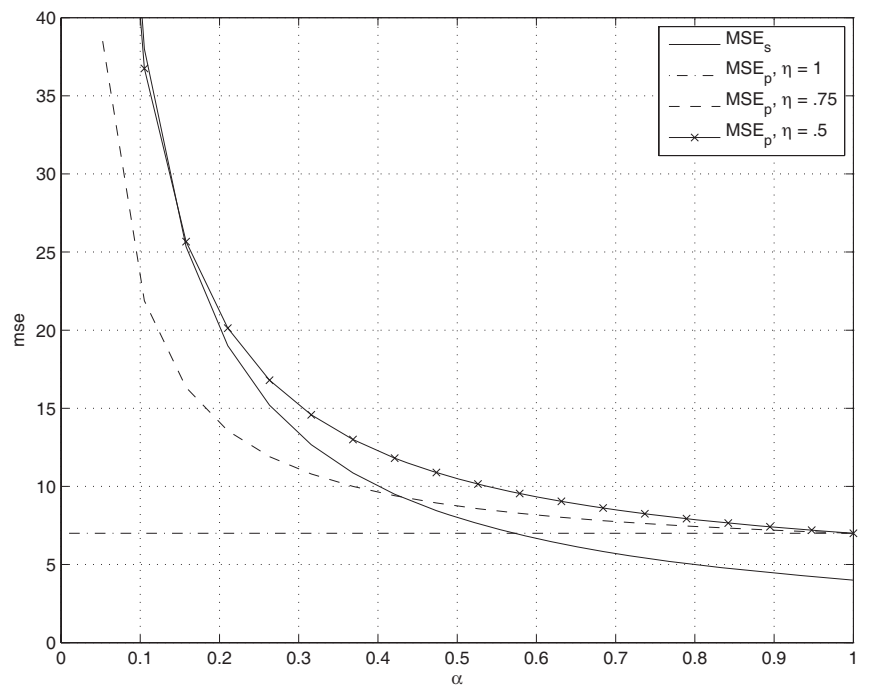

Fig. 4. $M S E_{s}$ and $M S E_{p}$ vs. $\alpha$ for various $\eta$.

results demonstrate sub-optimal pilot-power allocations for the primary and secondary users under various primary-channel stability scenarios, $\eta$.

For comparison, in Fig. 3 we plot the power settings $P_{1}$ and $P_{2}$ using the dedicated-relay-node criteria (10) discussed in Section II. These are plotted in Fig. 3 against the suboptimal MSE QoS rules (26) and (29), using $\delta_{s, r}^{2}=.001$ and $\delta_{r, d}^{2}=.001$. For a typical dedicated decode-and-forward relaying scheme, the relay would allocate $P_{1}=.408$ and $P_{2}=.372$. From Fig. 3 we note that the sub-optimal power allocation using the MSE rule tends toward the dedicatedrelay power allocation, $P_{2}$, as $\eta \rightarrow 0$. Additionally, we observe that as the channel becomes more stationary, i.e. as $\eta \rightarrow 1$, less pilot power is required to maintain a fixed channel estimate MSE for the primary-user, and $\alpha$ decreases under (26) accordingly.

Heuristically, we expect that frequent channel re-estimation will be required when the primary-channel is undergoing change, thus in this scenario the relay-node will be less inclined to sacrifice power for relay transmissions, and $\alpha$ will increase accordingly. Conversely, we expect that when channel conditions require less frequent channel estimation, the relay will behave altruistically and sacrifice energy for relay transmissions. Thus, in a typical power allocation policy $\alpha$ will be a monotonically decreasing function of $\eta$. This behavior is demonstrated in the MSE-based power allocation rule depicted in Fig. 3.

Next, we observe the general behavior of the channel estimate MSE for both the primary and secondary users as the pilot-power allocation $\alpha$ varies. In Fig. 4 a plot of the channel estimate MSE for the primary-user, $M S E_{p}$, and the secondary-user, $M S E_{s}$, is presented for the range $0 \leq \alpha \leq 1$, for fixed values of $\eta$. We expect that as $\alpha$ increases, the energy devoted to pilot signals used for channel estimation will also increase, and the MSE of the channel estimate for the secondary-user will decrease. In general, channel estimate MSE for both users will be a decreasing function of $\alpha$, which is demonstrated in Fig. 4. Also, when $\eta$ is exactly 1 the channel estimator is a perfect representation of the channel 


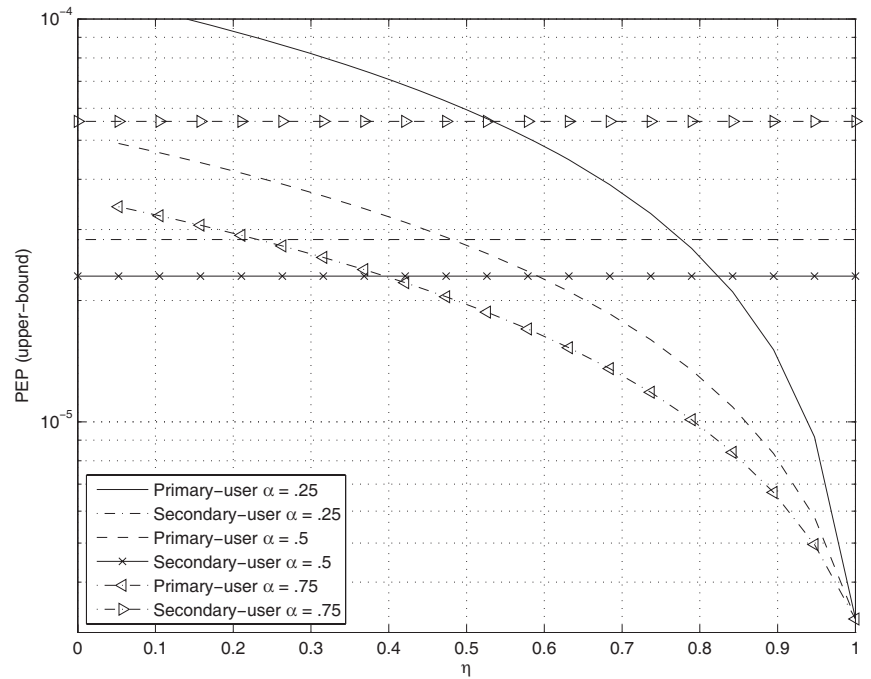

Fig. 5. $P E P$ vs. $\eta$ for fixed $\alpha$ - MSE rule.

with probability 1 , and the error of the channel estimate remains constant and invariant of the choice of $\alpha$.

Fig. 5 demonstrates the PEP of the primary and secondary users for fixed values of $\alpha$, with respect to $\eta$. The results presented here are for the values of $L_{t_{p}}=L_{r_{p}}=L_{r_{s}}=2$ and $P_{p}=1$. We note that as $\eta \rightarrow 1$ the channel becomes increasingly stationary, and the PEP for the primary-user decreases accordingly under the fixed MSE rule. Conversely, as it is assumed that the secondary-user is unconditionally required to preform channel estimation based on the pilot energy present, the PEP response for the secondary-user under the fixed MSE rules remains constant and independent of $\eta$, when $\alpha$ is fixed.

We now observe the behavior of a relay-node operating under the dynamic MSE-based power allocation rule (27). Fig. 6 demonstrates the PEP results of (13) and (14) using $\alpha=\alpha_{p_{M S E}}^{*}$, with respect to $\eta$, for various values of $T_{p}$. For comparison, PEP results for both users are also shown for the fixed power allocation policy $\alpha=0.5$. The best-effort behavior this system is readily discernible, as the primaryuser enjoys a general PEP advantage over the secondary-user. As the relay-node sacrifices energy for pilot signals used for channel estimation purposes, that is as $\eta \rightarrow 1$ in response to increasingly stationary channel conditions, the PEP of the primary-user improves as the PEP of the secondary-user degrades, for the values $T_{p}=3$ and $T_{p}=5$. This trend is demonstrated by the PEP offset between the primary and secondary-user PEP curves for $T_{p}=3$ and $T_{p}=5$. The behavior of the system when $T_{p}=7$ will be discussed shortly.

From Fig. 6 we note that as channel conditions become more deterministic, i.e. $\eta \rightarrow 1$, the PEP for the secondaryuser degrades, a curious result indeed. This phenomena may be explained by the increasingly worsened channel estimate a secondary-user would obtain as energy devoted to pilot signals is drastically decreased, since both the primary and secondary users use the same pilot signals for channel estimation. We observe that the PEP curve for the secondary-user is highly determined by the value chosen for $T_{p}$. As less model estimate MSE is permitted in the primary-user's channel estimate, i.e.

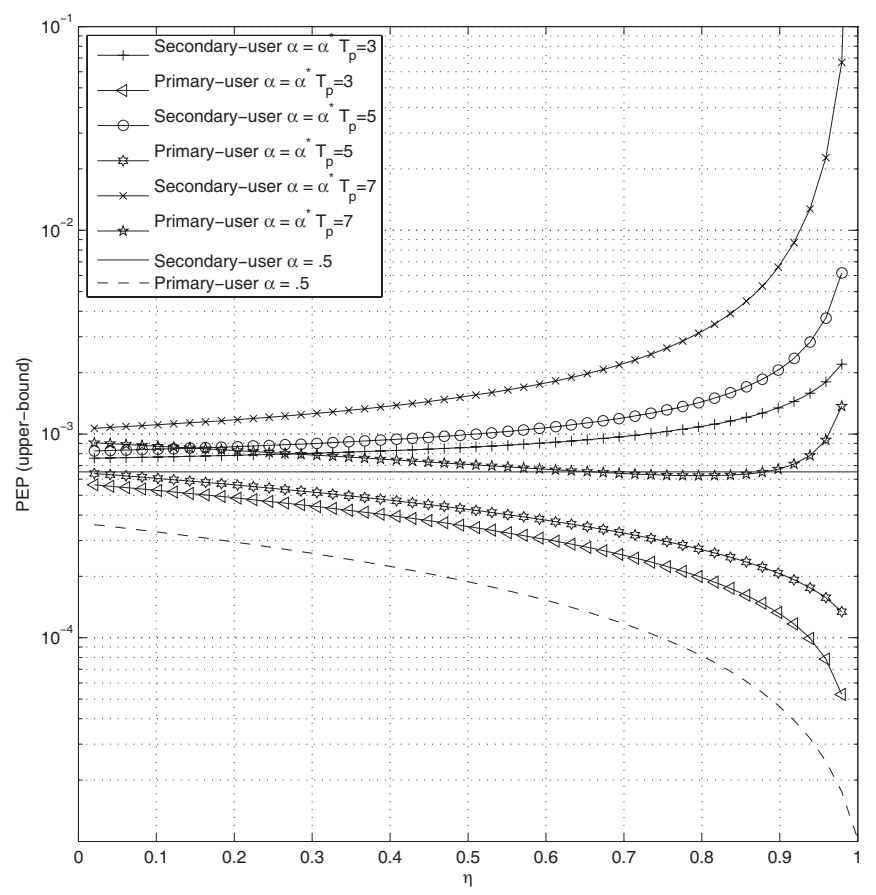

Fig. 6. PEP vs. $\eta$ for values of $T_{p}$ - MSE rule.

as value of the threshold $T_{p}$ is decreased, the primary-user becomes increasingly more conservative with the amount of energy it diverts from channel estimation devices resulting in a decreased PEP for the primary-user. Since the secondary-user is required to unconditionally use pilot energy for channel estimation independently of $\eta$, it will benefit from additional pilot energy when preforming channel estimation; however the signal strength of its data signal will suffer as the relay diverts less energy toward the relay services, and thus, PEP of the secondary-user degrades.

We observe that for larger values of $T_{p}$, as is the case $T_{p}=7$, increased model error severely degrades the general performance of both receivers resulting in detrimental effects for both users. In particular, we observe that as $\eta \rightarrow 1$ the PEP actually increases for both users when larger values of $T_{p}$ are used. We conclude that the value for $T_{p}$ must be carefully chosen with respect to the SNR experienced by both users under the MSE-based QoS rule (27), to ensure that sufficient pilot energy is retained. The main drawback of the MSEbased QoS rule is that the secondary-user cannot benefit from additional relay assistance when the primary communication mission becomes less difficult, i.e. as $\eta \rightarrow 1$, since the PEP for the primary-user decreases while the PEP for the secondaryuser dramatically increases, in this scenario.

In this motivating example we use identical channel SNRs for the primary and secondary users, that is $\sigma_{p}^{2}=\sigma_{s}^{2}$, and we have shown that an overall benefit to a secondary-user is obtainable in the form of useful relay bandwidth, without significant degradation of service to the primary-user. Thus, we have demonstrated that relay-diversity is achievable when a node with primary transmission responsibilities also employs cooperative relaying techniques, under the channel estimate MSE QoS rule. 


\section{Sub-optimal Power Allocation With Respect to PEP con- straint}

In the previous subsection, the sub-optimal power allocations for the best-effort relaying problem were presented for a power allocation policy that optimizes with respect to channel estimate MSE. We now consider the power allocation problem for relays that instead optimize with respect to a rule limiting the PEP experienced by the primary-user. From (19) and (21) the Chernoff upper-bound PEP expression under the two-state model can be expressed as

$$
\begin{aligned}
& P(\mathbf{d} \rightarrow \mathbf{e})_{\mathbf{H}_{p}(t)} \leq P(\mathbf{d} \rightarrow \mathbf{e} \mid \nu=0)_{\mathbf{H}_{p}(t)} P(\nu=0) \\
& +P(\mathbf{d} \rightarrow \mathbf{e} \mid \nu=1)_{\hat{\mathbf{H}}_{p}(t)} P(\nu=1) \\
& =\eta Q_{p}\left[R_{p}-(1-1 / \eta) S_{p}(\alpha)\right], \quad 0 \leq \alpha \leq 1
\end{aligned}
$$

where

$$
\begin{gathered}
Q_{p}=\left(\frac{1}{4 \sigma_{p}^{2}}\right)^{-L_{\Delta_{p}} L_{r_{p}}}\left(\prod_{i=1}^{L_{\Delta_{p}}} \lambda_{i_{p}}\right)^{-L_{r_{p}}}, \\
R_{p}=P_{p}^{-L_{\Delta_{p}} L_{r_{p}}}, \\
S_{p}(\alpha)=\left(\frac{1+\frac{\sigma_{s}^{2}}{\alpha}}{\frac{1}{N}\left(\frac{N}{P_{p}}+\frac{L_{t_{p}}}{\alpha}\right)}\right)^{-L_{\Delta_{p}} L_{r_{p}}} .
\end{gathered}
$$

The previous simplifications allow us to observe the behavior of sub-optimal power allocations with respect to the terms $Q$, $R$, and $S$ when manipulating $\alpha$ and $\eta$. For a fixed channel and antenna arrangement $L_{r_{p}}, L_{t_{p}}, \lambda_{i_{p}}$, and $\sigma_{p}^{2}$, we note that $Q, R$, and $S$ become constants with respect to a fixed $\alpha$. For a fixed $\eta$, we observe that the only term dependent on $\alpha$ is $S$, and all other aspects of the result are fixed for a power constraint $P_{p}$. We evaluate this system under the constraint that the relaynode must maintain a minimum QoS with respect to the PEP of its primary transmissions. The QoS constraint (11) for the primary-user minimizing $\alpha$ with respect to the PEP of (31) is simply

$$
G_{p}(\alpha, \eta)=\eta Q_{p}\left[R_{p}-(1-1 / \eta) S_{p}(\alpha)\right], \quad 0 \leq \alpha \leq 1 .
$$

Similarly, the constraint for the secondary-user from (14) has a QoS threshold determined by maximum allowable PEP. This constraint is a function of the relay's choice of $\alpha$, or exactly

$$
G_{s}(\alpha)=\left(\prod_{i=1}^{L_{\Delta_{s}}} \lambda_{i_{s}}\right)^{-L_{r_{s}}}\left(\frac{1+\frac{\sigma_{s}^{2}}{\alpha}}{\frac{4 \sigma_{s}^{2}}{N}\left(\frac{N}{P_{p}-\alpha}+\frac{L_{t_{s}}}{\alpha}\right)}\right)^{-L_{\Delta_{s}} L_{r_{s}}}
$$

The sub-optimal power allocation for the best-effort relay with respect to a maximum allowable PEP for the primary-user is found by solving (35) for $\alpha_{p_{P E P}}$, i.e.,

$$
\alpha_{p_{P E P}}^{*}=\frac{-P_{p}\left(L_{t_{p}}-\Psi\left(G_{p}, \eta\right) \sigma_{p}^{2}\right)}{N-P_{p} \Psi\left(G_{p}, \eta\right)}
$$

where

$$
\Psi\left(G_{p}, \eta\right)=\left(\frac{\eta P_{p}^{-L_{r_{p}} L_{\Delta_{p}}}-G_{p}\left(\frac{N}{4 \sigma_{p}^{2}}\right)^{L_{r_{p}} L_{\Delta_{p}}}}{\eta-1}\right)^{\frac{1}{L_{r_{p} L_{\Delta_{p}}}}} .
$$

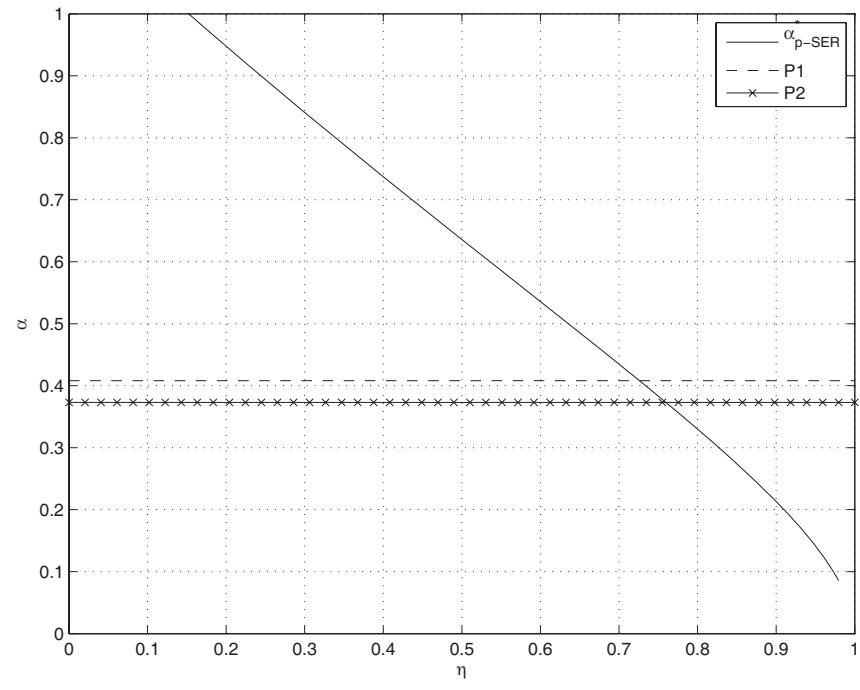

Fig. 7. $\alpha$ vs. $\eta$ for $G_{p}=1.4 e-5$ - PEP rule.

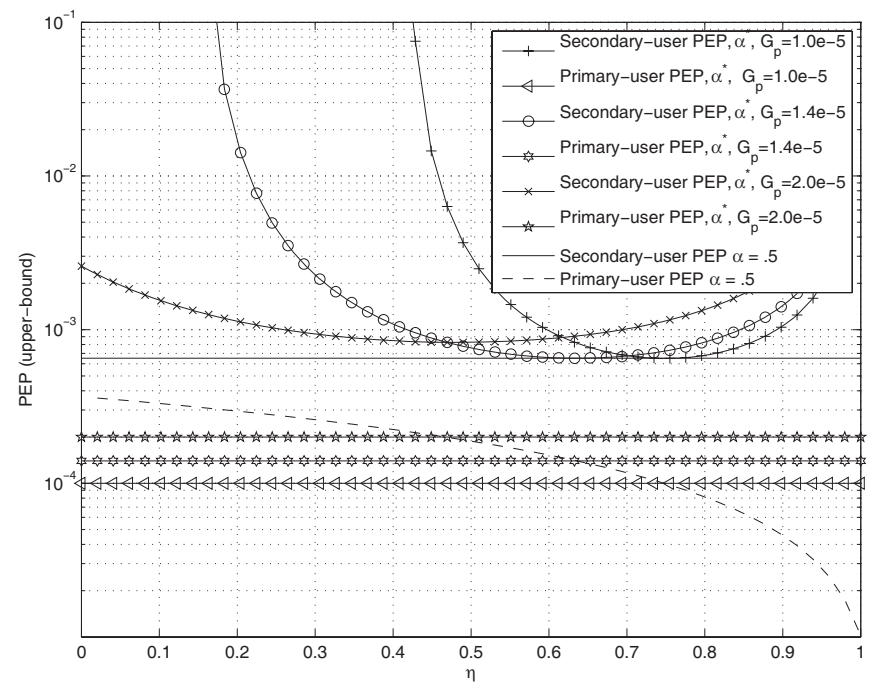

Fig. 8. PEP vs. $\eta$ for values of $G_{p}$ - PEP rule.

The PEP behavior using the PEP-based sub-optimal power application rule (37) with respect to $\eta$ is shown in Fig. 7 for the threshold value $G_{p}=1.4 e^{-5}$.

We have now proposed two QoS criteria for allocating power under the best-effort relay model, given as $\alpha_{p_{P E P}}^{*}$ and $\alpha_{p_{M S E}}^{*}$ derived in (27) and (37), respectively. If we compare the PEP behavior demonstrated in Fig. 7 with the MSE-based power allocation rule depicted in Fig. 3, we see that for (27) the acceptable power allocation range $0 \leq \alpha \leq 1$ is valid over a much wider range of $\eta$ for the MSE-based rule, when compared to the PEP-based rule (37), for the value of $G_{p}$ selected.

The PEP behavior of a relay-node operating under the power constraint (37) is shown in Fig. 8 according to (35) and (36), with respect to the channel estimate confidence coefficient $\eta$, for various values of $G_{p}$. As was demonstrated with the MSE-based power allocation rule, cooperative diversity gains are also obtainable using the PEP rule, as the relay diverts energy to secondary-user transmissions. The same phenomena of increased secondary-user PEP under high channel stationary 


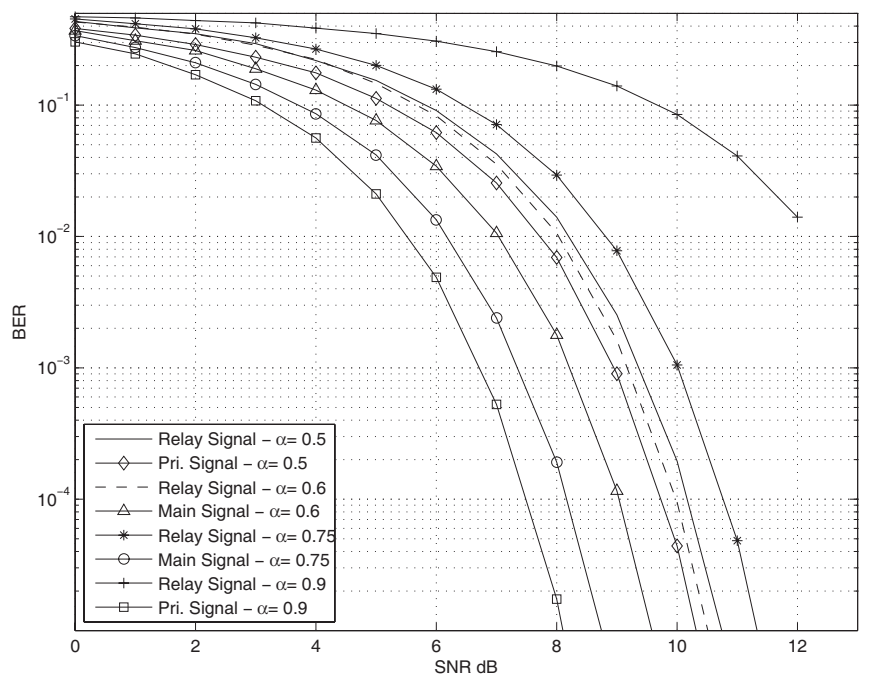

Fig. 9. BER for primary and secondary-users for various $\alpha$.

$(\eta \rightarrow 1)$ is apparent for the same reasons mentioned in Section III-B. As the relay diverts too much energy away from pilot signals, the PEP for the secondary-user suffers since the performance of this receiver requires sufficient pilot energy for channel estimation. Conversely, the primary-user enjoys an increasingly stationary channel with a high probability while its PEP remains the same. The salient difference between the MSE-based and PEP-based power allocation rules is demonstrated in their PEP behaviors: We expect that a power allocation rule optimizing with respect to specific maximum primary-user PEP threshold will exhibit a constant PEP response over all channel stationary states. This behavior is clearly discernible from Fig. 8, as PEP for the primary-user is constant-valued for the entire range $0 \leq \eta \leq 1$.

The best-effort behavior of the system under this rule is also apparent, as the primary-user consistently enjoys an PEP advantage over the secondary-user. The behavior of the PEP threshold $G_{p}$ is shown in Fig. 8 and may be compared to that of the MSE-based rule in Fig. 6. As $G_{p}$ increases, the relay will too readily divert energy from pilot signals and the PEP of the primary and secondary-users suffer accordingly. In general, decreasing $G_{p}$ has the effect of improving PEP for both the primary and secondary-users, at the cost of decreasing useful capacity for the best-effort channel when channel stability confidence is reduced (i.e. the PEP curve for the secondaryuser is shifted to the right). We conclude that the threshold $G_{p}$, like $T_{p}$, must also be carefully chosen with respect to the SNR experienced by the primary-user under the PEP-based QoS rule (37). In this example identical channel SNRs were used for both channels, demonstrating an overall benefit for the secondary-user using best-effort cooperative relaying.

\section{Simulation Results}

In this section we present simulation results for the proposed system for various values of $\alpha$, using the minimum meansquared channel estimator MMSE [17] and 2x2 Alamouti ST codes with $M=3$ and $N=L_{t_{p}}=2$. A block-stationary channel model was used with QPSK constellations and an ML symbol decoder. In Fig. 9, we plot the BER experienced by the

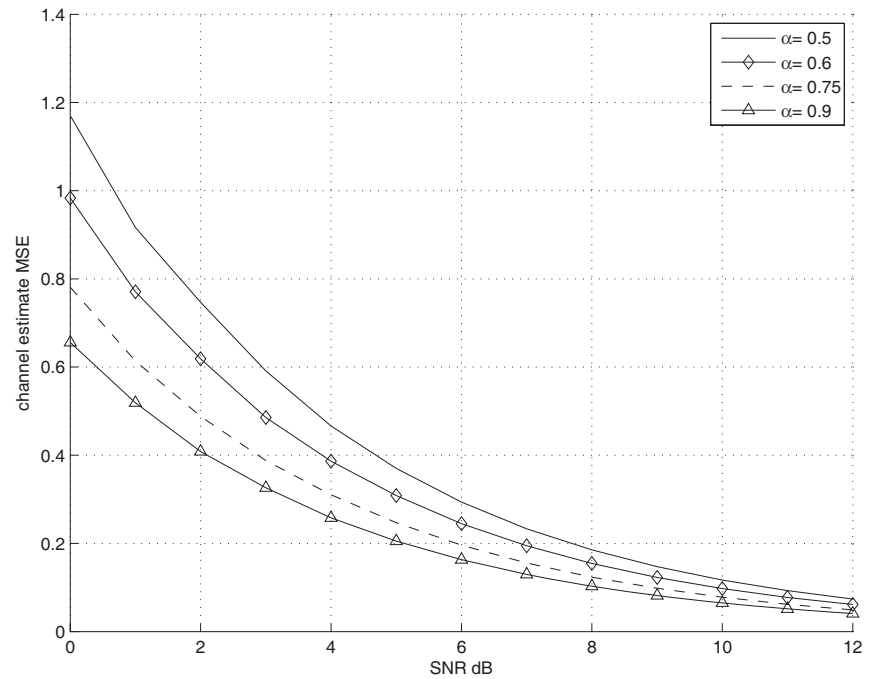

Fig. 10. Channel estimate MSE for various $\alpha$.

primary and secondary-users obtained through Monte Carlo MATLAB simulations using values of $\alpha$ equal to 0.5, 0.6, 0.75, and 0.9. In The BER in Fig. 9 is plotted vs. SNR, where SNR in this figure is defined the same as in Fig. 2. We observe that as power is retained for pilot signals the BER for the primary-user improves; however this improvement is achieved at the expense of decreased SNR for the secondary-user's data signal. We also observe that the BER for the secondary-user when $\alpha=0.6$ is better than the BER experienced at $\alpha=0.5$ and $\alpha=0.75$, suggesting that secondary-user BER is a convex function of $\alpha$ with a BER maximum somewhere between these two values. This behavior is due to the trade-off between data signal energy and pilot signal energy afforded to the secondary-user. A plot of the channel estimate MSE for both users is given in Fig. 10 vs. SNR, where SNR in this figure is defined the same as in Fig. 2. We observe that as the value of $\alpha$ increases more energy is allocated for channel estimation, thus the MSE of the receiver decreases accordingly.

We would like to note that the previous sections presented the behavior of the primary and secondary-nodes as a function of $\eta$ operating with a best-effort policy using two different power allocation rules. While these results are sub-optimal with respect to the primary-user, they fail to achieve optimal allocation with respect to the cooperative system. We now compare the results of the best-effort policy with a power allocation policy that attempts to maximize overall system capacity. The maximum-capacity QoS rule is defined by a policy that allocates power with respect to the primary and relay transmissions in a way that minimizes the sum of the BERs for both links.

The overall system BER is

$$
B E R_{t o t}=r_{p} P_{p}(\mathbf{d} \rightarrow \mathbf{e})+r_{s} P_{s}(\mathbf{d} \rightarrow \mathbf{e}),
$$

where $r_{p}$ and $r_{s}$ are the bits/code/Hz for the primary and secondary transmissions respectively, and $P_{p}(\mathbf{d} \rightarrow \mathbf{e}), P_{s}(\mathbf{d} \rightarrow \mathbf{e})$ are the PEP expressions for the primary and secondary-users respectively. A plot of the PEP for the overall system for various values of $\alpha$ is given for the case $r_{s}=r_{p}=.5 \mathrm{in} \mathrm{Fig.}$ 11. The results shown are for channel estimate confidence 


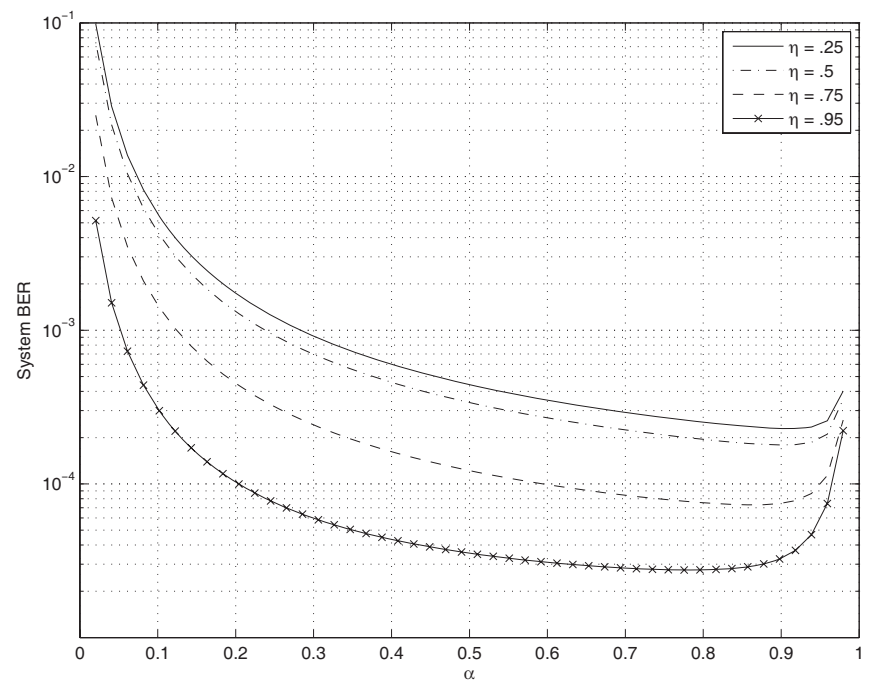

Fig. 11. Total System BER vs. $\alpha_{p}$ for various $\eta$.

coefficients $\eta=.25, \eta=.5, \eta=.75$, and $\eta=.95$, with $L_{r_{s}}=L_{r_{p}}=L_{t}=2$, with primary signal to noise ratio $S N R_{p}=13 \mathrm{~dB}$, the left hand side of (25), and secondary signal to noise ratio $S N R_{s}=20 \mathrm{~dB}$, the left hand side of (28). We note that Fig. 11 clearly demonstrates that an optimal value for $\alpha$ exists that minimizes the total BER for the cooperative system, and this value changes with respect to the channel stability $\eta$. Unfortunately, an analytical solution for this value is difficult to derive due to the large number of variables in (39). We note, however, that tractable analytical solutions for $\alpha$ exist for specific antenna configurations $L_{r_{s}}, L_{r_{p}}$ and, $L_{t}$ and fixed channel conditions $\lambda_{i_{p}}, \lambda_{i_{s}}, \Delta_{L_{p}}$, and $\Delta_{L_{p}}$, and the exploration of solutions to (39) under various scenarios remains future work.

\section{CONCLUSION}

We have demonstrated that cooperative diversity can be achieved through a best-effort delivery policy. In one example best-effort relaying scenario, energy scavenged from pilot signals was re-purposed for relay transmissions when channel conditions accommodate. It was demonstrated that in certain circumstances a node may sacrifice resources for relaying signals while maintaining a level of QoS for the primary-user, allowing the node to cooperate at its own discretion. Allowing nodes with primary communication missions to cooperate on a best-effort basis may lead to increased performance in cooperative communication systems, when compared to systems in which only dedicated-relays are allowed.

In deriving a sub-optimal power allocation policy, the MSE and PEP QoS rules were considered. It was demonstrated that the MSE QoS rule may yield lower PEP for the primary-user than the PEP-based QoS rule, for certain ranges of $\eta$, and that the MSE QoS rule yields acceptable relay service over a larger range of channel stationary conditions, when compared to the PEP-based rule. The drawback of the MSE-based QoS rule is that the secondary-user does not receive extra assistance from the relay when the primary communication mission becomes less difficult, i.e. as channel conditions become more stationary for the primary-user, since the PEP for the primaryuser increases and the PEP for the secondary-user decreases using this rule. Conversely, the PEP-based QoS rule provides a constant PEP for the primary-user over all values of $\eta$ while providing extra assistance to the secondary-user via decreased PEP; however it yields acceptable relay performance over a small range of $\eta$ only.

\section{REFERENCES}

[1] K. J. R. Liu, A. Sadek, W. Su, and A. Kwasinski, Cooperative Communications and Networking. Cambridge University Press, 2009.

[2] J. N. Laneman and G. W. Wornell, "Distributed space-time coded protocols for exploiting cooperative diversity in wireless networks," IEEE Trans. Inf. Theory, vol. 49, no. 10, pp. 2415-2525, Oct. 2003.

[3] E. Larsson and B. R. Vojcic, "Cooperative transmit diversity based on superposition modulation," IEEE Commun. Lett., vol. 9, no. 9, pp. 778780, Oct. 2005

[4] T. M. Cover and A. A. E. Gamal, "Capacity theorems for the relay channel," IEEE Trans. Inf. Theory, vol. 25, no. 5, Sep. 1979.

[5] R. Chen, J. Park, and J. Reed, "Defense against primary user emulation attacks in cognitive radio networks," IEEE J. Sel. Areas Commun., vol. 26, no. 1, pp. 25-37, Jan. 2008.

[6] M. Herdin, N. Czink, H. Ozcelik, and E. Bonek, "Correlation matrix distance, a meaningful measure for evaluation of non-stationary MIMO channels," in IEEE Vehicular Technology Conference, vol. 1, pp. 136140 , June 2005.

[7] J. Wallace and M. Jensen, "Time-varying MIMO channels: measurement, analysis, and modeling," IEEE Trans. Antennas Propag., vol. 54, pp. 3265-3273, Nov. 2006.

[8] B. M. H. B. Hassibi, "How much training is needed in multiple-antenna wireless links?" IEEE Trans. Inf. Theory, vol. 49, pp. 951-963, Apr. 2003.

[9] C. Pirak, Z. J. Wang, K. J. R. Liu, and S. Jitapunkul, "A data-bearing approach for pilot-embedding frameworks in space-time coded MIMO systems," IEEE Trans. Signal Process., pp. 3966-3979, Oct. 2006.

[10] W. Su, Z. Safar, and K. J. R. Liu, "Full-rate full-diversity spacefrequency codes with optimum coding advantage," IEEE Trans. Inf. Theory, pp. 229-250, Jan. 2005.

[11] A. Sendornaris, E. Erkip, and B. Aazhang, "User cooperation diversity, part I: system description," IEEE Trans. Commun., vol. 51, pp. 19271938, Nov. 2003

[12] J. N. Laneman, D. N. C. Tse, and G. W. Wornell, "Cooperative diversity in wireless networks: efficient protocols and outage behavior," IEEE Trans. Inf. Theory, vol. 50, no. 12, pp. 3062-3080, Dec. 2004.

[13] S. Haykin, "Cognitive radio: brain-empowered wireless communications," IEEE J. Sel. Areas Commun., vol. 23, no. 2, pp. 201-220, 2005.

[14] N. Goergen, K. J. R. Liu, and T. C. Clancy, "Best-effort cooperative communication without dedicated relays," IEEE International Conference on Acoustics, Speech, and Signal Processing, Mar. 2010.

[15] C. Pirak, Z. J. Wang, K. J. R. Liu, and S. Jitapunkul, "Adaptive channel estimation using pilot-embedded data-bearing approach for MIMOOFDM systems," IEEE Trans. Signal Process., vol. 54, no. 12, Dec. 2006.

[16] W. Su, A. Sadek, and K. J. R. Liu, "Cooperative communication protocols in wireless networks: performance analysis and optimum power allocation," Wireless Personal Commun., vol. 44, pp. 181-217, Jan. 2008.

[17] S. Alamouti, "A simple transmitter diversity scheme for wireless communications," IEEE J. Sel. Areas Commun., vol. 16, pp. 1414-1458, Oct. 1998.

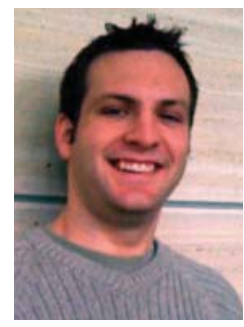

Nate S. Goergen ( $\left.\mathrm{S}^{\prime} 03\right)$ is a $\mathrm{Ph} . \mathrm{D}$. candidate in the Electrical and Computer Engineering Department, University of Maryland, College Park. He received the B.S. in Electrical Engineering from Rose-Hulman Institute of Technology in 2004, and the M.S. degree in Electrical and Computer Engineering from the University of Maryland, College Park in 2010. He was awarded the DoD S.M.A.R.T Scholarship in 2007. His research interests include cognitive radio, signal processing, and physical layer security of wireless signals. His current research is in watermarking approaches for wireless communications. 


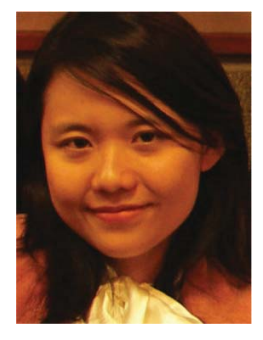

W. Sabrina Lin (M'06) received the Ph.D. degree with the Electrical and Computer Engineering Department, University of Maryland, College Park, where she is a Research Associate. She received the B.S. and M.S. degrees in Electrical Engineering from National Taiwan University in 2002 and 2004, respectively. Her research interests are in the area of information security and forensics, multimedia signal processing and multimedia social network analysis. She received the University of Maryland Future Faculty Fellowship in 2007.

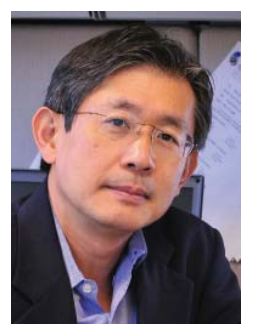

K. J. Ray Liu (F'03) is named a Distinguished Scholar-Teacher of University of Maryland, College Park, in 2007, where he is Christine Kim Eminent Professor of Information Technology. He serves as Associate Chair of Graduate Studies and Research of Electrical and Computer Engineering Department and leads the Maryland Signals and Information Group conducting research encompassing broad aspects of wireless communications and networking, information forensics and security, multimedia signal processing, and biomedical engineering.

Dr. Liu is the recipient of numerous honors and awards including IEEE Signal Processing Society Technical Achievement Award and Distinguished Lecturer. He also received various teaching and research recognitions from University of Maryland including university-level Invention of the Year Award; and Poole and Kent Senior Faculty Teaching Award and Outstanding Faculty Research Award, both from A. James Clark School of Engineering. An ISI Highly Cited Author in Computer Science, Dr. Liu is a Fellow of IEEE and AAAS.
Dr. Liu is President-Elect and was Vice President-Publications of the IEEE Signal Processing Society. He was the Editor-in-Chief of IEEE Signal Processing Magazine and the founding Editor-in-Chief of EURASIP Journal on Advances in Signal Processing.

His recent books include Cognitive Radio Networking and Security: A Game Theoretical View (Cambridge University Press, 2010); Behavior Dynamics in Media-Sharing Social Networks (Cambridge University Press (to appear)); Handbook on Array Processing and Sensor Networks (IEEEWiley, 2009); Cooperative Communications and Networking (Cambridge University Press, 2008); Resource Allocation for Wireless Networks: Basics, Techniques, and Applications (Cambridge University Press, 2008); UltraWideband Communication Systems: The Multiband OFDM Approach (IEEEWiley, 2007); Network-Aware Security for Group Communications (Springer, 2007); and Multimedia Fingerprinting Forensics for Traitor Tracing (Hindawi, 2005).

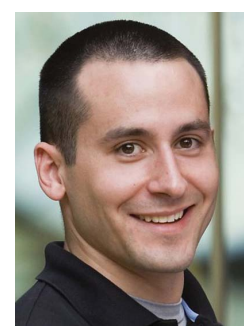

T. Charles Clancy (S'02-M'06-SM'10) is the Associate Director of the Ted and Karyn Hume Center for National Security and Technology at Virginia Tech, where he leads the university's educational and research efforts in national security. Prior to joining Virginia Tech, Dr. Clancy led a number of wireless research programs at the Laboratory for Telecommunications Science, at the University of Maryland, emphasizing development in commodity use of software-defined radio. Dr. Clancy's research interests are in the security of wireless communications, particularly spectrum access and waveform robustness.

Dr. Clancy received his $\mathrm{PhD}$ in Computer Science from the University of Maryland, College Park, MS in Electrical Engineering from the University of Illinois, Urbana-Champaign, and BS in Computer Engineering from the RoseHulman Institute of Technology, Terre Haute, IN. He is a Senior Member of the IEEE. 